\title{
Shells in the Magellanic System
}

\author{
Snežana Stanimirović $\dagger$ \\ Radio Astronomy Lab, UC Berkeley, 601 Campbell Hall, Berkeley, CA 94720, USA
}

\begin{abstract}
The Magellanic System harbors > 800 expanding shells of neutral hydrogen, providing a unique opportunity for statistical investigations. Most of these shells are surprisingly young, 2-10 Myr old, and correlate poorly with young stellar populations. I summarize what we have learned about shell properties and particularly focus on the puzzling correlation between the shell radius and expansion velocity. In the framework of the standard, adiabatic model for shell evolution this tight correlation suggests a coherent burst of star formation across the whole Magellanic System. However, more than one mechanism for shell formation may be taking place.
\end{abstract}

Keywords. ISM: bubbles, ISM: structure, Magellanic Clouds

\section{Introduction}

Numerous studies over the past three decades have shown that shell-like structures dominate the interstellar medium (ISM) in many galaxies. This sculpturing of the ISM, mainly assumed to be due to star-formation activity, must have an imprint on many physical processes (e.g. the transport of radiation, heating and cooling etc.). In the traditional scenario, shells are expected to be reservoirs of hot gas and are the sole-source of the hot, intercloud medium. At the same time, these dynamic features are sites of local energy depositions in the ISM which contribute significantly to the total energy budget. Another important role shells have is in providing the connection between galactic disks and halos. Shells grow in the disks, expand, and the largest ones can reach sizes larger than the disk scale height. When this happens shells open up and vent hot gas into the halo.

Despite significant observational efforts, the exact mechanism(s) for shell formation is still not fully understood. Similarly, the late stages of shell evolution have not been explored. The most accepted model for shell formation, the "Standard Model" (Weaver et al. 1977; McCray \& Kafatos 1987), views shells as products of combined effects of stellar winds and supernovae. Numerous observational puzzles however motivated other suggestions. For example, several other types of powering sources were suggested, the most exotic ones being pulsars and gamma-ray bursts. Several proposed mechanisms do not even require the existence of a central energy source. These range from the collision of high-velocity clouds (HVCs) with a galactic disk, through results of the general ISM turbulence, with or without gravitational and thermal instabilities, the ram pressure stripping, flaring of radio lobes, to the complex new conceptual designs of the ISM in the form of an elastic polymer interwoven with magnetic field lines (Cox 2005). This list is obviously long, and surprisingly keeps growing steadily.

In this paper we focus on shells discovered in the Magellanic System: the Small Magellanic Cloud (SMC), the Large Magellanic Cloud (LMC), and the Magellanic Bridge (MB). More than 800 shell-like structures were found and cataloged in high-resolution neutral hydrogen (HI) observations of these three environments. All observations were conducted

$\dagger$ Present address: Department of Astronomy, University of Wisconsin, 475 North Charter Street, Madison, WI 53706, USA email: sstanimi@astro.wisc.edu 
Table 1. Summary of shell properties: Number of HI shells, the min/max range of shell radii, the min/max range of shell expansion velocities, the estimated mean dynamic age, and power-law slopes of the shell size and expansion velocity distribution functions.

\begin{tabular}{|c|c|c|c|c|c|c|}
\hline & $\mathrm{N}$ & $\begin{array}{l}R_{\mathrm{s}} \\
(\mathrm{pc})\end{array}$ & $\begin{array}{c}V_{\exp } \\
\left(\mathrm{km} \mathrm{s}^{-1}\right)\end{array}$ & $\begin{array}{c}\left\langle T_{\mathrm{s}}\right\rangle \\
(\mathrm{Myr})\end{array}$ & $\alpha_{\mathrm{r}}$ & $\alpha_{\mathrm{v}}$ \\
\hline $\mathrm{SMC}$ & 509 & $20-800$ & $2-33$ & 5.7 & $-2.5 \pm 0.2^{a}$ & $-2.2 \pm 0.3^{a}$ \\
\hline MB & 163 & $10-200$ & $2-20$ & 6.2 & $-3.6 \pm 0.4$ & $-2.6 \pm 0.6$ \\
\hline LMC & $124(54)^{b}$ & $50-620$ & $6-36$ & 4.8 & $-2.5 \pm 0.4$ & $?$ \\
\hline
\end{tabular}

with the Australia Telescope Compact Array (ATCA) and were complemented with the short-spacing data from surveys with the Parkes telescope. These data sets sample a wide and continuous range of spatial scales, providing unique opportunities for finding and studying large samples of expanding shells. It is important to stress that the three systems we investigate here probe very different interstellar environments. The SMC is a dwarf irregular, gas-rich galaxy with a large line-of-sight depth, the LMC is a dwarf disk galaxy with traces of spiral structure and a higher star formation rate, while the $\mathrm{MB}$ is a column of gas between the two galaxies that was formed as a result of tidal interactions. In Section 2 we summarize the most important properties of HI shells in the Magellanic System, and address their implications in Section 3. In Section 4 we point out recent observational work in the domain of the late stages of shell evolution, and then summarize in Section 5.

\section{Summary of observational properties}

\subsection{Shells in the $S M C$}

More than 500 expanding shells have been cataloged in the SMC (Staveley-Smith et al. 1997; Stanimirović et al. 1999; Hatzidimitriou et al. 2005). Table 1 lists the typical shell radius $\left(R_{\mathrm{s}}\right)$ and the expansion velocity $\left(V_{\mathrm{exp}}\right)$. Curiously, as noted first by Staveley-Smith et al. (1997), all SMC shells appear to have a very similar dynamic age, $T_{\mathrm{s}} \sim 5 \mathrm{Myr}$. This was interpreted as evidence for a single, coherent, and global burst of star formation in the SMC. The volume occupied by all these shells is large, about $40 \%$ of the whole SMC, implying a very bubbly, or a 'Swiss Cheese'-like morphology. Estimating the fraction of HI mass occupied by shells is more difficult as we need an estimate of the local ambient density. If we assume that the local ambient surface density for all shells is $0.01 \mathrm{M}_{\odot}$ $\mathrm{pc}^{-2}$, we arrive at a total mass fraction that is about $20 \%$.

Recently, Hatzidimitriou et al. (2005) searched for the remnant stellar population associated with the HI shells by using all available catalogues of the young stellar populations. About 450 shells were found to correlate with one or more stellar objects, while 59 shells do not correlate with any of the known stellar objects. We will refer further to these two classes of shells as "non-empty" and "empty", respectively. The surprising result is that properties of "non-empty" and "empty" are almost indistinguishable. There are no morphological differences between the two groups. "Empty" shells appear smaller and with a lower expansion velocity than "non-empty" ones, however this is primarily a selection effect. Both types also show an almost linear correlation between the shell radius and expansion velocity. Spatially, "empty" shells are found primarily in remote places on the outskirts of the HI distribution. Of course, finding similar shells in the central parts would be impossible. Several "empty" shells with high luminosity appear loosely 
clustered and possibly connected; these objects may belong to an old chimney. The rest of the empty shells, however, do not have any special location or association.

Hatzidimitriou et al. (2005) also derived the shell size and expansion velocity distribution functions, $N\left(R_{\mathrm{S}}\right)$ and $N\left(V_{\text {exp }}\right)$. For both types of shells $N\left(R_{\mathrm{S}}\right)$ and $N\left(V_{\exp }\right)$ can be fitted with a power-law function, $N\left(R_{\mathrm{s}}\right) \propto R_{\mathrm{s}}^{\alpha_{\mathrm{r}}}$ and $N\left(V_{\exp }\right) \propto V_{\exp }^{\alpha_{\mathrm{v}}}$. The slopes $\alpha_{\mathrm{r}}$ and $\alpha_{\mathrm{v}}$ agree within their uncertainties for the two types of shells. In the framework of the standard model, based on whether all shells were formed in a single burst or in a continuous manner, and by assuming either a single input mechanical luminosity function (MLF) for all shells or a power-law function, we can predict $\alpha_{\mathrm{r}}$ and $\alpha_{\mathrm{v}}$ and then compare these values with what we get from observations. This was first shown by Oey \& Clark (1997). The positive slope of the $R_{\mathrm{s}}-V_{\exp }$ correlation, and the fact that $\alpha_{\mathrm{r}} \approx \alpha_{\mathrm{v}}$ $(\approx-2.2 \pm 0.2$ for "non-empty shells $)$, point to the case of a single burst of shell formation and a power-law input MLF. It is puzzling though that the same arguments apply to "empty" shells as well. If the "empty" shells are > 10 Myr old, and this could be the reason why we do not find their corresponding stellar population, then for them to fit on the same $R_{\mathrm{s}}-V_{\exp }$ relation would require a significant and concerted re-acceleration.

\subsection{Shells in the Magellanic Bridge}

Muller et al. (2003) cataloged 163 shells in the MB, applying criteria somewhat tighter than in the case of the SMC shells (for example, they do not include incomplete large shells in their catalog; this results in shell sizes being biased towards smaller shells). Shell sizes and expansion velocities are given in Table 1. The estimated mean dynamic age is $T_{\mathrm{s}}=6.2 \mathrm{Myr}$, with a standard dispersion of 3.4 Myr. Muller et al. (2003) cross-correlated their shell catalog with the catalog of OB associations by Bica \& Schmitt (1995) and found that about $60 \%$ of shells do not have corresponding OB associations. Also, while the mean dynamic shell age is about $6 \mathrm{Myr}$, the mean age of OB associations is several times larger, 10-25 Myr. Although the MB is a tidal remnant of the interactions between the LMC and the SMC, shells in the MB are primarily spherical and without obvious signs of distortions or tidal stretching.

\subsection{Shells in the $L M C$}

There are 101 giant $\left(R_{\mathrm{S}}<360 \mathrm{pc}\right)$ and 23 super-giant $\left(R_{\mathrm{S}}>360 \mathrm{pc}\right)$ shells in the LMC (Kim et al. 1999). Shell radii are in the range 50-620 pc, while shell expansion velocities are in the range $6-36 \mathrm{~km} \mathrm{~s}^{-1}$. Interestingly, while the expansion velocity is systematically higher in the LMC than in the SMC, about one half of all LMC shells appear to have stalled, with $V_{\exp }=0$. The mean dynamic age is $4.9 \mathrm{Myr}$, which is again younger than the age of corresponding $\mathrm{OB}$ associations ( $>10 \mathrm{Myr}$ ). Giant shells in the LMC also follow the almost linear $R_{\mathrm{s}}-V_{\text {exp }}$ relation, while the super-giant shells deviate from this trend. Kim et al. (1999) also found a poor spatial correlation between shells and OB associations. The shell size distribution is $N\left(R_{\mathrm{S}}\right) \propto R_{\mathrm{S}}^{-2.5}$, and is similar to that for the SMC shells.

\section{Putting it all together}

To summarize, the properties of all shells in the SMC, the MB, and the LMC show striking similarities: the dynamic age, tight $R_{\mathrm{s}}-V_{\exp }$ relation, statistical properties, poor correlation with stellar populations, and dynamic ages being younger than those of $\mathrm{OB}$ associations. And yet, the three environments in which these shells formed and evolved are drastically different! To emphasize this, we plot the dynamic shell age as a function of Right Ascension in Figure 1 and include all (> 800) shells found in the Magellanic System. 


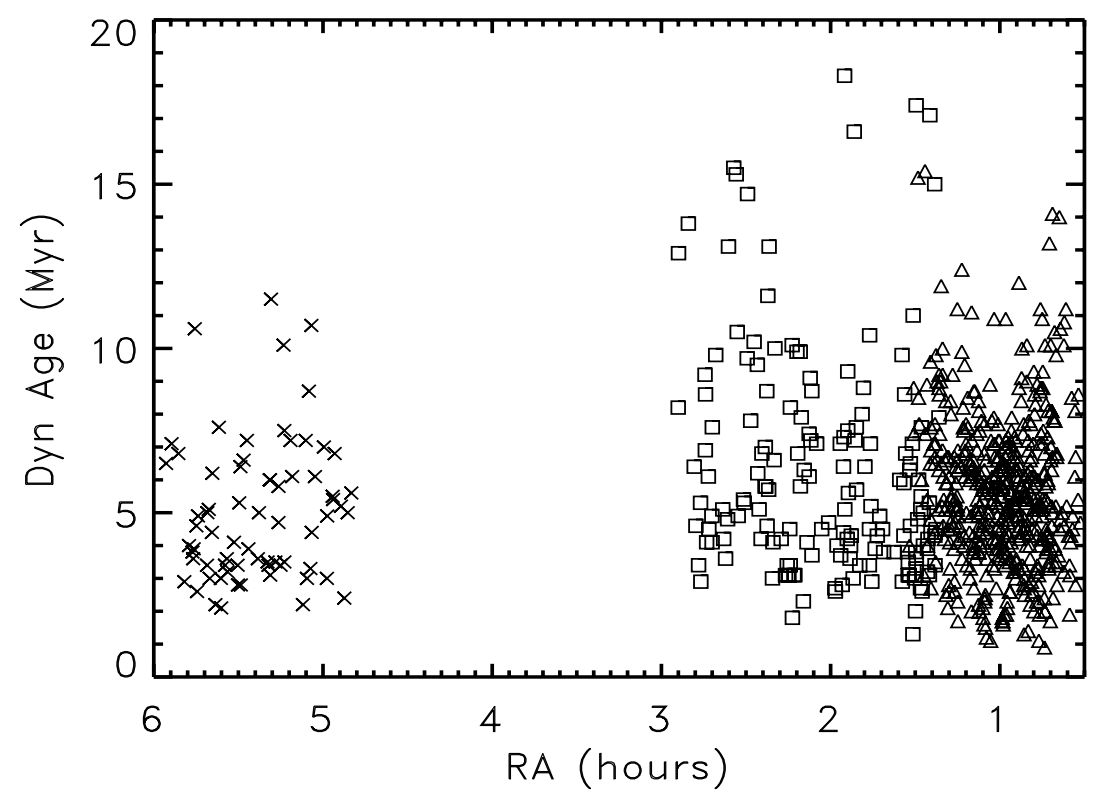

Figure 1. The estimated dynamic age for shells in the SMC (triangles), the MB (squares), and the LMC (crosses).

This distribution appears uniform and there are no obvious discontinuities between the SMC and the MB. It is also apparent that the SMC and the MB have a few shells older than the majority of the LMC shells. Figures 1 and 2 also show that, on average, the shell size increases from the SMC, through the MB, to the LMC. This is primarily a selection effect, however. For example, Muller et al. (2003) discuss their lack of large shells in the $\mathrm{MB}$ and explain that this is mainly due to their more stringent criteria when identifying shells.

In Figure 2 we plot all shells on the $R_{\mathrm{s}}-V_{\text {exp }}$ diagram. It is very curious how all shells appear to follow the same, tight relation, being nested between the solid lines that mark $T=2 \mathrm{Myr}$ and $T=10 \mathrm{Myr}$, when viewed within the standard model. In the case of our Galaxy, Ehlerová \& Palouš (2005) did not find a correlation between the shell radius and expansion velocity, although even there it looks like larger shells have a larger $V_{\text {exp }}$. In the framework of the standard model, the tight age spread could be interpreted as a result of a recent star formation burst across the whole Magellanic System about 5 Myr ago. However, this is difficult to reconcile with the star formation history of the Clouds. In the case of the SMC, Harris \& Zaritsky (2004) estimated ages of $>5 \times 10^{6}$ stars and found the closest peak in star formation $60 \mathrm{Myr}$ ago. It is also interesting to note that on the olderage side, the cut-off in the shell distribution (Figure 2) is sharper than on the youngerage side. This may be suggestive of an enhanced shell destruction/fragmentation after $\sim 10$ Myr. However, this is significantly shorter than the typical predicted shell lifetime of 30-50 Myr (Dove 2000; Wünsch \& Palouš 2001).

The $R_{\mathrm{s}}-V_{\text {exp }}$ diagram obviously suffers from some selection effects and is biased towards younger objects. Large shells are hard to distinguish observationally as they are often fragmented and also could be seen as a superposition of several smaller shells. In the case of the SMC, Zaritsky \& Harris (2004) showed that 10 to $70 \%$ of all stars 


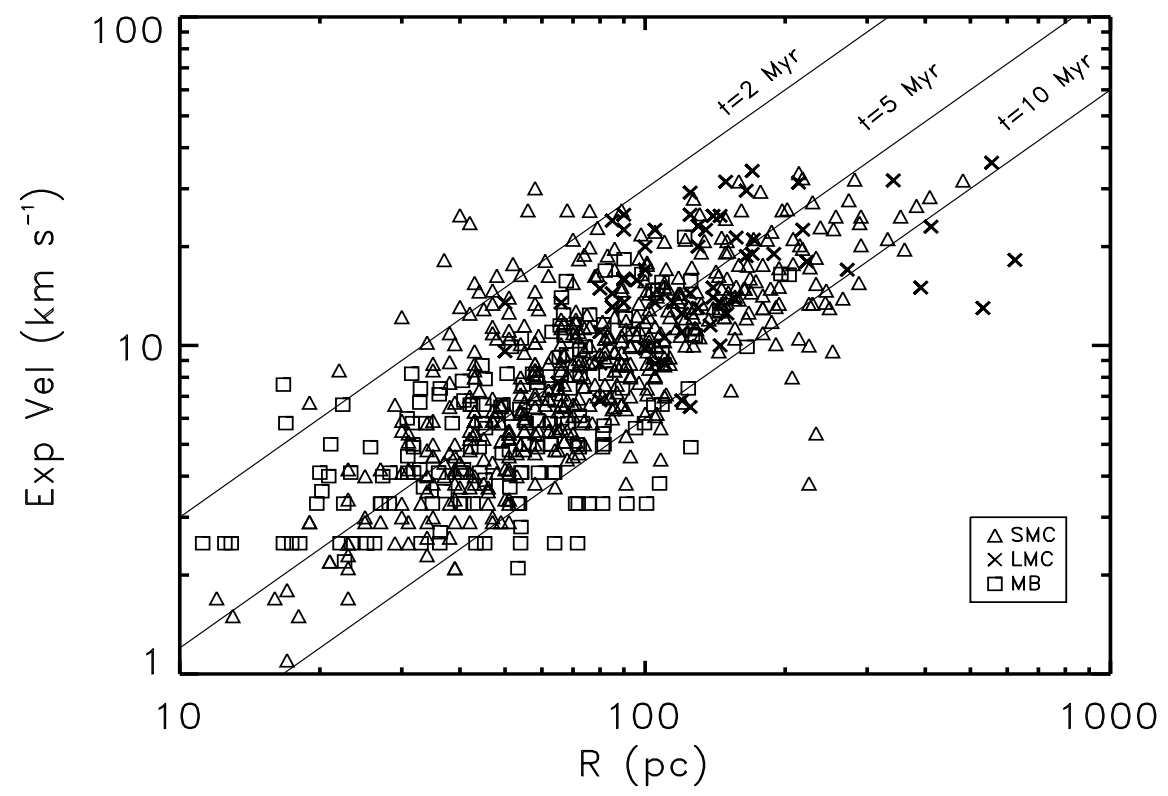

Figure 2. Size and expansion velocity for shells in the SMC (triangles), the LMC (crosses), and the MB (squares). The solid lines represent dynamic ages of 2, 5, and 10 Myrs, respectively, in the framework of the standard model.

could have formed through tidal triggering. Tidal triggering would obviously affect shell formation, and probably even shell evolution. The degree with which these processes are constrained and coordinated across the Magellanic System still needs to be explored. Alternatively, there may be one or more additional shell-formation processes taking place. Obviously, understanding the role of environmental effects (e.g. tidal flows, the turbulent ISM, interactions between shells, magnetic field etc.) is crucial for further advancement.

\section{What happens to shells at a very late stage of their evolution?}

We would now like to draw attention to numerous HI clouds found recently in the interface region between the disk and the halo of our Galaxy (Lockman 2002; Stil et al. 2005; Stanimirović et al. 2006). These clouds are small, 5-20 pc in size, cold $(<400$ to $1000 \mathrm{~K}$ ), and often kinematically follow the Galactic disk but at a velocity that is offset by $10-20 \mathrm{~km} \mathrm{~s}^{-1}$ from that of the bulk HI emission. The clouds do not appear to prefer particular regions in the Galaxy, their distribution is most likely radially extended. The origin of these clouds is not clear. There are several possibilities including Galactic fountains and the accretion of extragalactic gas. An alternative possibility, however, is that these clouds are fragments of expanding shells. There are several pieces of evidence that point in this direction. For example, a Galactic chimney, GSH242-03+37 shows small, discrete HI clouds that appear associated with shell caps (McClure-Griffiths et al. 2006). Lockman, Pidopryhora, \& Shields (2006) found a large plume-like structure which has numerous clouds. Stanimirović et al.. (2006) found that clouds are embedded in large filamentary structures and morphologically resemble cold clouds that form in simulations of dynamically triggered instabilities (e.g. Audit \& Hennebelle 2005). One way of testing the hypothesis that these newly-discovered clouds are shell fragments is to derive the 
cloud mass spectrum and compare it with theoretical predictions from shell fragmentation. For example, Wünsch \& Palouš (2001) predict that the mass spectrum for shell fragments is a power-law with $d N / d M \propto M^{-1.4}$. The mass spectrum of observed clouds will be easily derived in the near future as several large HI surveys are underway with the Arecibo, Parkes and Green Bank telescopes that are particularly suited for finding these clouds.

\section{Summary and open questions}

There are $\sim 800$ shells in total in the SMC, the MB and the LMC. At least 1/10 of SMC shells are devoid of stellar counterparts, but surprisingly have properties similar to those of "non-empty" shells. Large similarities in shell properties across the Magellanic System, and especially the tight correlation between the shell radius and expansion velocity, are puzzling and may be highlighting the importance of tidal interactions for both shell formation and evolution. Alternative processes for shell formation and external effects may be also playing important roles. The late stages of shell evolution are finally being addressed observationally, and detailed theoretical attention is highly desirable in this area. And finally, there is the Magellanic Stream, a starless tidal tail that provides the perfect opportunity to quantify the importance of shell formation processes without a powering source. This is an obvious future project!

\section{Acknowledgements}

I would like to thank the conference organizers for inviting me to participate in this highly stimulating and enjoyable conference. I would also like to thank Carl Heiles, one of the pioneers of shell exploration, for numerous inspiring discussions, and Jacco van Loon for many insightful comments. This work was partially supported by NSF grants AST 04-06987 and 00-97417.

\section{References}

Audit, E. \& Hennebelle, P. 2005, A\& A 433, 1

Bica, E.L.D. \& Schmitt, H.R. 1995, ApJS 101, 41

Cox, D.P. 2005, ARAA 43, 337

Dove, J.B., Shull, J.M. \& Ferrara, A. 2000, ApJ 531, 846

Ehlerová, S. \& Palouš, J. 2005, A\&GA 437, 101

Hatzidimitriou, D., Stanimirović, S., Maragoudaki, F., Staveley-Smith, L., Dapergolas, A. \& Bratsolis, E. 2005, MNRAS 360, 1171

Kim, S., Dopita, M.A., Staveley-Smith, L. \& Bessell, M.S. 1999, AJ 118, 2797

Lockman, F.J., Pidopryhora, Y. \& Shields, J.C. 2006, this conference.

McClure-Griffiths, N.M., Ford, A., Pisano, D.J., Gibson, B.K., Staveley-Smith, L., Calabretta, M.R., Dedes, L. \& Kalberla, P.M.W. 2006, ApJ 638, 196

McCray, R. \& Kafatos, M. 1987, ApJ 317, 190

Muller, E., Staveley-Smith, L., Zealey, W. \& Stanimirović, S. 2003, MNRAS 339, 105

Stanimirović, et al. 2006, ApJ in press (astro-ph/0609137)

Stanimirović, S., Staveley-Smith, L., Dickey, J.M., Sault, R.J. \& Snowden, S.L. 1999, MNRAS 302,417

Staveley-Smith, L., Sault, R.J., Hatzidimitriou, D., Kesteven, M.J. \& McConnell, D. 1997, MNRAS 289, 225

Weaver, R., McCray, R., Castor, J., Shapiro, P. \& Moore, R. 1977, ApJ 218, 377

Wunsch, R. \& Palouš, J. 2001, A\&A 374, 746 


\section{Discussion}

Y.-H. CHU: If there was a burst of star formation 5 Myr ago to form all these shells, there should be late $\mathrm{O}$ and early $\mathrm{B}$ stars remaining in the shells. However, when we searched for such stars from uv observations with IUE/HST/FUSE, we couldn't find any within the larger shells in the LMC. They couldn't have all been formed from a single burst of star formation 5 Myr ago.

StANIMIROVIC: This is just one more argument against interpreting all these shells as being caused by stellar winds/ SNe. 